\title{
Segmentation of the right ventricle in MRI images using a dual active shape model
}

\author{
ISSN 1751-9659 \\ Received on 3rd February 2016 \\ Accepted on 25th March 2016 \\ E-First on 27th May 2016 \\ doi: 10.1049/iet-ipr.2016.0073 \\ www.ietdl.org
}

\author{
Hossam El-Rewaidy 1,2, El-Sayed Ibrahim ${ }^{3}$, Ahmed S Fahmy ${ }^{1,2} \bowtie$ \\ ${ }^{1}$ Systems and Biomedical Engineering, Cairo University, Giza, Egypt \\ ${ }^{2}$ Center for Informatics Science, Nile University, Giza, Egypt \\ ${ }^{3}$ Department of Radiology, University of Michigan, Ann Arbor, USA \\ 凶-mail: asfahmy@nu.edu.eg
}

\begin{abstract}
Active shape models (ASM) showed to have potential for segmenting the right ventricle (RV) in cardiac magnetic resonance images (MRIs). Nevertheless, the large variability and complexity of the RV shape do not allow for concisely capturing all possible shape variations among patients and anatomical cross-sections. Noticeably, the latter increases the number of iterations required to converge to a proper solution and reduces the segmentation accuracy. In this study, the authors propose a new ASM framework that can model the RV shape in short-axis cardiac MRI images. In this framework, the RV contour is split into two simpler segments, septal (SP) and free wall, whose shape variations are independently modelled using two separate (dual) ASM models. The contour splitting is done at the location of the RV insertion points into the SP wall. Further, instead of using the conventional Procrustes method, the RV contours are aligned using the Bookstein coordinate transformation, which uses the RV insertion points as landmarks to linearly align the RV contours. The results from a dataset of 14 patients show that the proposed framework outperforms the conventional ASM framework and can model complex RV shape variation with more accuracy and in less iteration steps.
\end{abstract}

\section{Introduction}

Assessment of the structure and function of the right ventricle (RV) from cardiac magnetic resonance images (MRIs) plays an important role in diagnosing and monitoring a number of cardiovascular diseases, including pulmonary hypertension, cardiomyopathy, myocardial infarction, and congenital heart disease [1-4]. In a typical study, a stack of parallel short-axis crosssections of the heart is acquired, from which the RV volume is computed to estimate important clinical parameters, such as the RV ejection fraction $[2,4]$. A necessary step for such analysis is the delineation of the RV boundaries in the acquired images at the different cardiac phases. The automation of this process is challenging due to the complex shape of the RV, presence of trabecular muscles, irregular RV shape in certain diseases (e.g. severe pulmonary hypertension), image artefacts, and low tissueto-blood contrast (especially at the apical cross-sections due to the accentuated partial volume artefacts) [5]. This challenging problem has been investigated by a number of researchers using a number of different image processing techniques [5-19].

Generally, the ventricular boundary detection techniques can be classified as either atlas-based (probabilistic [20], statistical [20], or geometrical [21]), intensity-based [22], and model-based techniques [23-30]. While the atlas-based techniques are appealing, their implementation in clinical practice is limited by the need for extensive manual segmentation during the training process and the possibility of missing important cases during the training phase that could limit the atlas's capability of identifying similar cases during implementation. The intensity-based techniques are easier to implement; however, they are limited by the image quality, e.g. signal-to-noise ratio (SNR) and presence of artefacts, and the accumulation of tracking error, e.g. in the case of non-rigid image registration. The model-based analysis techniques provide a powerful approach for fast and accurate evaluation of the heart images. By customising anatomical and functional models of the heart to images from an individual subject, information about the heart shape and function can be obtained, which is helpful for studying normal ranges of variation in the population and differences in pathological cases. Compared with model-free approaches, the model-based techniques have the advantage that they provide information about the expected shapes of the structures of interest, which makes it easier to identify these structures in images with low SNR or in the presence of imaging artefacts. Further, the structures of interest can be described with a small number of parameters, and all image analysis and shape modelling steps can be conducted in a common framework. Modelbased analysis techniques have been implemented for evaluating global [31, 32] and regional [23, 26] heart function, and for investigating new descriptors of cardiac function [27-30].

Among the different techniques used for RV segmentation, active shape models (ASM's) showed to have a strong potential due to their ability to model the complex shape of the RV [15-19]. Basically, ASM's detect the cardiac contour by minimising an energy function that measures the difference between the model and image data. For two-dimensional (2D) RV segmentation, an ASM model is built for each cross-section by capturing the mean shape and principal modes of variations of the RV contours present in a training dataset. The latter is constructed from manually extracted RV contours [33, 34]. Nevertheless, the ASM technique is challenged by the extensive shape variability of the RV across different slices, timeframes, and patients. Especially, the latter does not allow for succinct representation, i.e. using a small set of modes of variations, of possible RV shapes.

In this work, we propose a modified ASM framework that can be used to efficiently capture the variations among the RV shapes. Two contributions have been included in this work. First, the RV contour is split into two segments: free (FR )wall and septal (SP) segments, where a separate ASM is built for each segment. The second contribution in this work is the normalisation of all the contour segments using the Bookstein transformation, rather than the conventional Procrustes alignment. As will be shown later, these two modifications allow for succinct representation of the RV contours, which leads to more accurate segmentation results. It is worth mentioning that the current work is based on some preliminary study presented in [35]. 


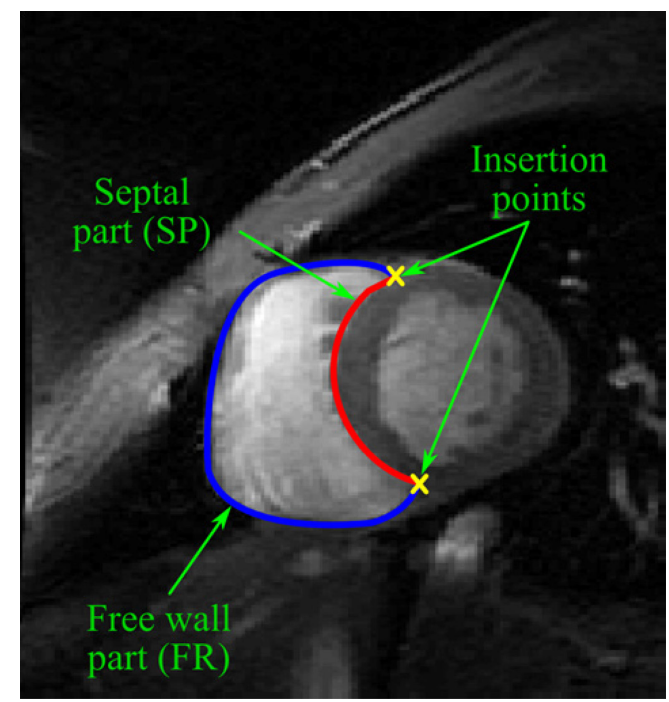

Fig. $1 R V$ contour is divided into two segments (SP and FR wall) in each short-axis image

\section{Methods}

\subsection{Conventional active shape modelling}

Conventional 2D ASM is based on constructing the shape model from a set of contours delineating the object boundaries in a set of training images. A fixed set of $n$ landmark boundary points, e.g. $\left\{\left(x_{1}, y_{1}\right),\left(x_{2}, y_{2}\right), \ldots,\left(x_{n}, y_{n}\right)\right\}$, are selected from each contour in the training set. Then, these training sets of points are aligned to minimise the variance in distance between the corresponding landmark points. This step is essential for proper model formation as it reduces the contributions from non-specific shape variations caused by, for example, affine transformation. The Procrustes alignment method is commonly used to align the contours in the training set $[19,24,33]$ using proper affine transformations. The $x$ and $y$-coordinate values of each point on a given aligned contour, $i$, are stacked to form a vector $\boldsymbol{x}$ of length $2 n$, i.e.

$$
\boldsymbol{x}_{i}=\left[x_{1}, x_{2} \ldots, x_{n}, y_{1}, y_{2} \ldots, y_{n}\right]^{\mathrm{T}} .
$$

Then, the mean, $\overline{\boldsymbol{x}}=(1 / n) \sum_{i=1}^{n} \boldsymbol{x}_{i}$, and the covariance, $\boldsymbol{S}=(1 /(n-1)) \sum_{i=1}^{n}\left(\boldsymbol{x}_{i}-\overline{\boldsymbol{x}}\right)\left(\boldsymbol{x}_{i}-\overline{\boldsymbol{x}}\right)^{\mathrm{T}}$, of the training contours are calculated and the shape model is represented by the following equation:

$$
\boldsymbol{x}=\overline{\boldsymbol{x}}+\boldsymbol{P} \cdot \boldsymbol{b},
$$

where $\boldsymbol{P}$ is a matrix of size $2 n \times k$ whose columns are the first $k$ Eigenvectors of the covariance matrix, and $\boldsymbol{b}$ is a vector of the shape parameters. The value of $k$ is determined as the smallest number of modes of variations that capture a predetermined percentage of the shape variance ( $=98 \%$ in this work) [33].

\subsection{Contour splitting}

To simplify the geometric complexity of the RV contours, each RV contour in the training dataset is split into two segments: the SP wall segment, $C_{\mathrm{sp}}$, and the FR wall segment, $C_{\mathrm{fr}}$, as shown in Fig. 1. Anatomically, the choice of the insertion points to split the

Table 1 Complexity metrics (mean $\pm S D$ ) of whole contours and individual segments

\begin{tabular}{lccc}
\hline Metric $^{a}$ & Complete RV & FR part & SP part \\
\hline notches & $0.5 \pm 0.05$ & $0.27 \pm 0.068$ & $0.23 \pm 0.12$ \\
ampl & $0.043 \pm 0.0078$ & $0.0017 \pm 0.002$ & $0.003 \pm 0.003$ \\
conv & $0.2013 \pm 0.03$ & $0.0115 \pm 0.011$ & $0.04 \pm 0.053$ \\
\hline
\end{tabular}

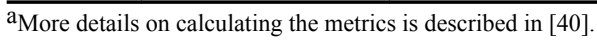

contour is based on fact that these two points are the anatomical junctions representing the location where the muscle of the RV is inserted into the left ventricle. That is, the shape variation of the FR wall during contraction, i.e. at the different cardiac phases, is independent from that of the SP wall [36-38]. Geometrically, the operation of splitting the contour at the insertion points reduces its complexity. Table 1 shows the complexity metrics (proposed by Brinkhoff et al. [39]) of the entire contour and the individual segments of all RV contours in the dataset. As can be seen in the table, all the complexity metrics are significantly reduced by splitting the contour. In the training phase, this process is performed semi-automatically, where the user is prompted, after drawing the RV contour, to select the two insertion points of the $\mathrm{RV}$ into the SP wall. Each contour segment is modelled separately, yielding a dual ASM model

$$
\begin{gathered}
\boldsymbol{x}^{\mathrm{sp}}=\overline{\boldsymbol{x}}^{\mathrm{sp}}+\boldsymbol{P}^{\mathrm{sp}} \boldsymbol{b}^{\mathrm{sp}}, \\
\boldsymbol{x}^{\mathrm{fr}}=\overline{\boldsymbol{x}}^{\mathrm{fr}}+\boldsymbol{P}^{\mathrm{fr}} \boldsymbol{b}^{\mathrm{fr}},
\end{gathered}
$$

To ensure continuity of the final segmentation results, an overlap (5\% of the contour points) between the two segments is maintained to allow the fusion of the two segments into a single smooth RV contour. By the end of the segmentation process, the two parts (SP and FR) are merged together using a B-Spline algorithm of order 3 to obtain a smooth contour at the cut points.

\subsection{Alignment in the Bookstein coordinates}

In this work, the Bookstein method is used to align the training contours [40]. The Bookstein method allows taking advantage of the manually selected insertion points in both model building, or training, phase as well as the segmentation phase. This can result in a more reasonable alignment of the contours because, first, it uses the line extending between the two insertion points as an orientation reference for rotating the training contours. This has advantage of properly align the contours in diseased conditions where the RV contours do not have a distinguished pose; i.e. of rounded shapes. The latter usually represents a challenge for the Procrustes method, which estimates the rotation angle that yields the minimum distance among the contours irrespective of the proper anatomical orientation. Another feature of the Bookstein method is that it implicitly scales the contours based on the distance between the two insertion points. This gives an advantage during the test phase, over the Procrustes method, because the mean contour is properly scaled to fit the size of the RV to be segmented. Another advantage of the Bookstein alignment is that it constrains the RV contour to pass through the two insertion points throughout the iterative segmentation process as will be discussed later. This prevents the contour, while searching for the best match of the appearance model, from taking arbitrary orientation, position, and scale.

Given the contour from the training set, the two insertion points, $\left(q_{1 x}, q_{1 y}\right)$ and $\left(q_{2 x}, q_{2 y}\right)$, are manually selected and transformed into points $(0,0)$ and $(1,0)$, respectively, in the Bookstein coordinates. The remaining contour points, $\left(c_{x}, c_{y}\right)$, are then transformed to their corresponding points, $\left(p_{x}, p_{y}\right)$, in the Bookstein coordinates using the following equations:

$$
\begin{aligned}
& p_{x}=\frac{\left(c_{x}-q_{1 x}\right) \times d_{x}+\left(c_{y}-q_{1 y}\right) \times d_{y}}{d_{x}^{2}+d_{y}^{2}}, \\
& p_{y}=\frac{\left(c_{y}-q_{1 y}\right) \times d_{x}-\left(c_{x}-q_{1 x}\right) \times d_{y}}{d_{x}^{2}+d_{y}^{2}},
\end{aligned}
$$

where $d_{x}=\left(q_{2 x}-q_{1 x}\right)$ and $d_{\mathrm{y}}=\left(q_{2 \mathrm{y}}-q_{1 \mathrm{y}}\right)$. It is worth noting that the inverse of (4) can be numerically solved to transform back a given point from the Bookstein coordinates to its original location in the image space. In order to achieve that, the trust region dogleg method for unconstrained optimisation is used to get the inverse Bookstein transformation [41] (See Fig. 2). That implies finding 

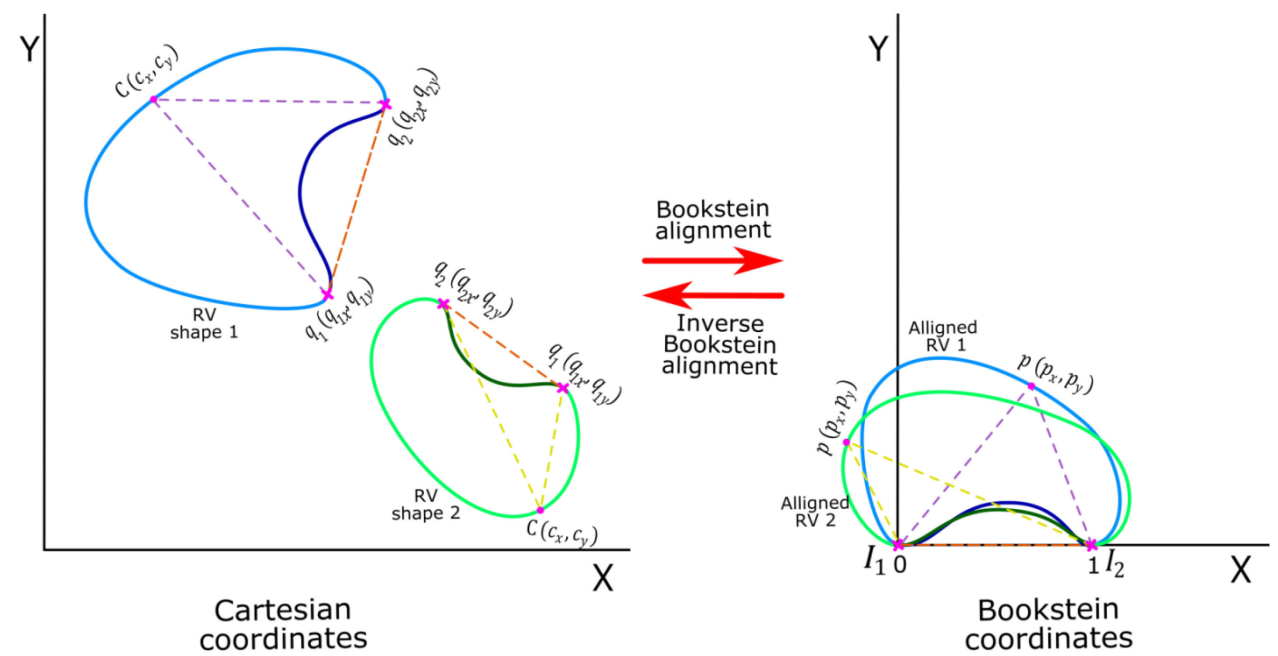

Fig. 2 Transformation of the $R V$ shape to the Bookstein $2 D$ coordinates can be performed in two steps: (i) registering the two insertion points, $\boldsymbol{q}_{1}$ and $\boldsymbol{q}_{2}$ to points 0 and 1 on the $x$-axis; and (ii) normalising each point on the original $R V$ shape with respect to the distance between the two insertion points. The $R V$ shape can be transformed back in the Cartesian coordinates by reversing the Bookstein transformation

the optimal positions of the landmark points by iteratively minimising the following cost function:

$$
C_{\text {opt }}=\arg \min _{C} F\left(C \mid p, q_{1}, q_{2}\right) \text {, }
$$

where (see (6))

\subsection{Dataset and training}

To test and validate the proposed framework, a database contains 840 images from the York University dataset of short-axis cardiac MRI images [42]. The images' size is $256 \times 256$ pixels with pixel size between 0.9 and $1.5 \mathrm{~mm}$. The dataset covers the whole cardiac cycle (20 frames per cycle) at three cross-sectional levels (basal, mid-cavity, and apical slices) from 14 different subjects. This yielded a total of $280 \mathrm{RV}$ shapes for each cross-sectional level. The dataset has been randomly divided into two subsets: a training set of 168 images that form $56 \mathrm{RV}$ shapes (about $20 \%$ of the whole dataset selected from 9 subjects) and a testing set of 672 images that form $224 \mathrm{RV}$ shapes. The ground truth is defined by manually delineating all RV boundaries in the dataset.

For each contour, 60 landmarks (contour points) at equal distances are used to represent the shape of that RV. Furthermore, two insertion points for each contour are included to form the baseline of the Bookstein alignment and to define the ends of the SP and FR parts. Both parts of the RV shapes in the training set were aligned separately with respect to their insertion points using 2D Bookstein coordinates, as mentioned before. Principal component analysis was then applied to the aligned shapes to estimate the mean shape for every part. The first 8 and 15 modes of variation (i.e. Eigen vectors) were selected to represent about 98 and $95 \%$ of the variance in the training set for the FR wall and SP parts, respectively.

For each contour point, $m$, a grey-level appearance model is built. First, the image is obtained by sampling the intensity along a line perpendicular to the contour and passes through this point. The length of intensity profile, $l$, was chosen to be 17 pixels centred at the contour point. For the entire set of training images, the mean intensity profile is obtained, $\overline{\boldsymbol{g}}_{m}$, as well as the covariance matrix. The first $k$ eigenvectors of the covariance matrix, corresponding to $98 \%$ of total variation, are used to represent the modes of variations matrix, $\boldsymbol{Q}_{\boldsymbol{m}}$, of size $=l \times k$. That is, the appearance model is given by the following equation:

$$
\boldsymbol{g}_{m}=\overline{\boldsymbol{g}}_{m}+\boldsymbol{Q}_{\boldsymbol{m}} \cdot \boldsymbol{h},
$$

where $\boldsymbol{h}$ is a $k \times 1$ vector representing the appearance parameters.

\subsection{Segmentation of the RV}

Fig. 3 shows a flowchart of the main steps applied to delineate the $\mathrm{RV}$ from a given input image. The same iterative implementation of the conventional ASM described previously by Ginneken et al. [18] is used in this work. First, the two RV insertion points, $I_{\mathrm{t} 1}$ and $I_{\mathrm{t} 2}$, are selected and used to inversely transform the mean contour, $\overline{\boldsymbol{x}}$, from the Bookstein coordinates to the image coordinates using the inverse of (4). The mean contour is then iteratively evolved, point-by-point, such that the image intensity profile in the neighbourhood of each contour point matches the profile captured by the appearance model. For example, given any contour point, the image intensity profile along a line passing through this point perpendicular to the evolved contour is obtained, $\boldsymbol{g}_{\boldsymbol{m}}$. Then, the iterative search algorithm continues to minimise the error function given by the following equation:

$$
e=\boldsymbol{D}^{-1 / 2} \cdot \boldsymbol{Q}_{\boldsymbol{m}} \cdot\left(\boldsymbol{g}_{\boldsymbol{m}}-\overline{\boldsymbol{g}}_{m}\right),
$$

where $\boldsymbol{D}$ is a diagonal matrix whose entries are the eigenvalues corresponding to the eigenvectors of the matrix $\boldsymbol{P}$. After evolving all of the contour points, the resulting contour is transformed to the Bookstein coordinates and projected on the shape model as in (8). The latter guarantees that the contour belongs to the subspace of all training RV shapes captured by the model. Finally, the resulting contour is transformed back to the image space and the process is repeated. The algorithm is then terminated after a pre-specified number of iterations ( $=20$ in this work).

Since conventional ASM uses only one point to set the initial location of the mean contour while the proposed model uses two points, the latter might have an advantage over the former. Therefore, to allow for fair comparison, the two RV insertion points were used to improve the initial orientation and scaling of the conventional ASM model, as shown in Fig. 4. First, assume that the location of the two insertion points on the mean RV contour are $I_{m 1}$ and $I_{m 2}$. Then, for forming the initial contour, a given point, $p(x, y)$, on the mean shape is transformed to point, $p^{\prime}(x$, $y)$, using the following equation:

$$
\begin{aligned}
F\left(C \mid p, q_{1}, q_{2}\right)=[ & \left(c_{x}-q_{1 x}\right) \times d_{x}+\left(c_{y}-q_{1 y}\right) \times d_{y}-p_{x} \times\left(d_{x}^{2}+d_{y}^{2}\right), \\
& \left.\left(c_{y}-q_{1 y}\right) \times d_{x}-\left(c_{x}-q_{1 x}\right) \times d_{y}-p_{y} \times\left(d_{x}^{2}+d_{y}^{2}\right)\right]
\end{aligned}
$$




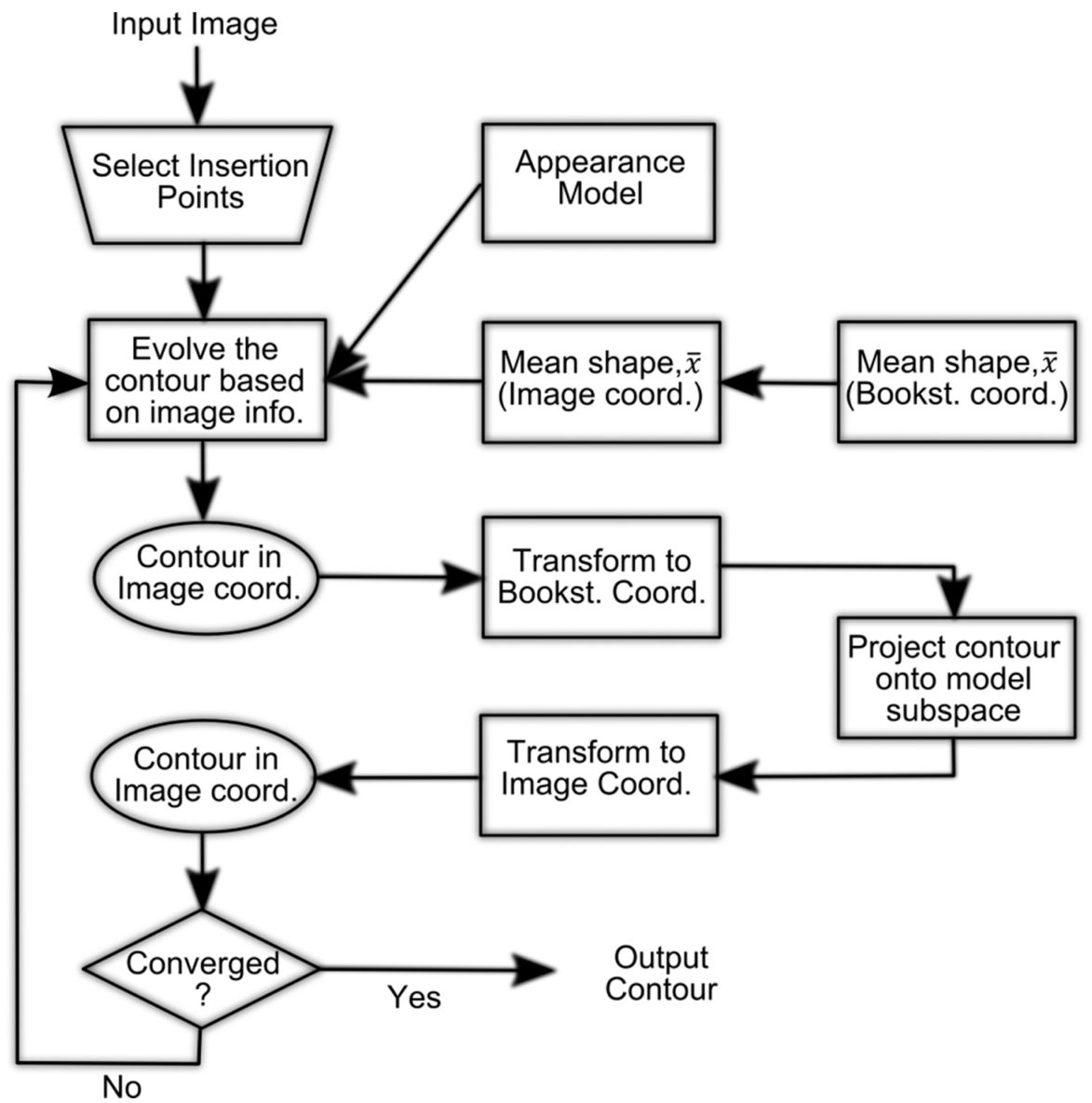

Fig. 3 Flowchart for RV segmentation using the proposed ASM framework, the same framework can be used for either FW or SP parts

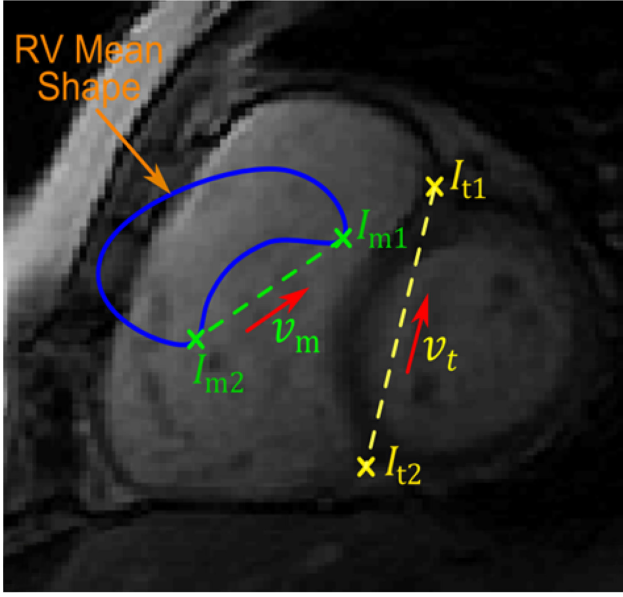

a

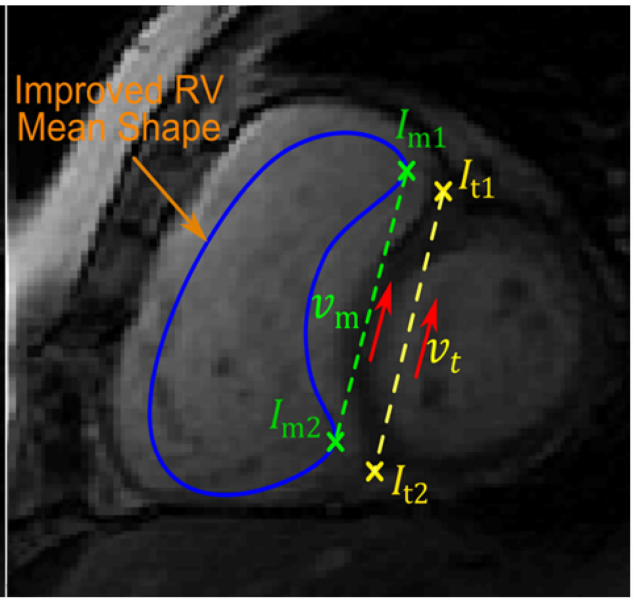

$b$

Fig. 4 Improved initial placement of the RV mean shape in conventional ASM, where the location of the two RV insertion points is used to estimate proper scaling, rotation, and translation parameters to form the mean shape

(a) Initial RV shape without improvements, (b) Initial RV shape with improvements

$$
\begin{aligned}
& p^{\prime}(x, y)=\alpha\left[\begin{array}{cc}
\cos (\theta) & -\sin (\theta) \\
\sin (\theta) & \cos (\theta)
\end{array}\right]\left(p(x, y)-\bar{x}_{\text {centre }}\right)+ \\
& \bar{x}_{\text {centre }}+d
\end{aligned}
$$

where $\alpha$ is a scaling factor, equal to $I_{\mathrm{t} 1}-I_{\mathrm{t} 2} / I_{m 1}-I_{m 2}, \bar{x}_{\text {centre }}$ is the centre-of-mass of the mean shape, $\theta$ is the angle between the two vectors $\left(I_{\mathrm{t} 1}-I_{\mathrm{t} 2}\right)$ and $\left(I_{m 1}-I_{m 2}\right)$, and $d$ is the approximate location of the RV centre point.

\subsection{Testing and validation}

The final contours extracted by the proposed ASM method are evaluated using the manually extracted contours as a reference. Three error measures are used to validate the results. The first measure is the mean absolute distance (MAD) [43]. The MAD is a local distance measure that calculates the average absolute distance between the estimated contour, $C^{\text {est }}$, and every point, $p_{i}$, on the manually delineated contour (ground truth)

$$
\mathrm{MAD}=\frac{1}{n} \sum_{i=1}^{n}\left|d\left(p_{i}, C^{\mathrm{est}}\right)\right|
$$


where $d(. .$.$) is the minimum Euclidean distance between the points$ $g_{i}$ and contour $C^{\text {est }}$ and, $n$ is the number of points on the contour. The second error measure used in this work is the Hausdorff distance [44], which measures the maximum distance between the estimated contour, $C^{\text {est }}$ and the reference contour, $C^{\text {ref }}$. This can be formulated as

$$
\begin{aligned}
& \text { Hausdorff }\left(C^{\text {est }}, C^{\text {ref }}\right)=\max \left(\max _{i}\left\{\mathrm{~d}\left(p_{i}, C^{\text {est }}\right)\right\}, \max _{j}\left\{\mathrm { d } \left(q_{j},\right.\right.\right. \\
& \left.\left.\left.C^{\text {ref }}\right)\right\}\right),
\end{aligned}
$$

where $q_{j}$ is the $j$ th point on $C^{\text {est }}$ and $p_{i}$ is the $i$ th point on $C^{\text {ref }}$. It is worth noting that these two measures, MAD and Hausdorff, are calculated in millimetre in our calculations for the segmentation errors. The third error measure used in this work is the Dice index [45], which measures the mean overlapping, or intersection, between the areas enclosed by the estimated contour, $C^{\text {est }}$, and that enclosed by the ground truth contour, $C^{\text {ref }}$

$$
\text { Dice }=2 \frac{\left|C^{\mathrm{ref}} \cap C^{\mathrm{est}}\right|}{\left|C^{\mathrm{ref}}\right|+\left|C^{\mathrm{est}}\right|}
$$

The Dice index ranges from 0 to 1 and, and unlike the MAD and Hausdorff measures, its value increases with improved segmentation accuracy.

\section{Results and discussion}

Table 2 shows the average [mean \pm standard deviation (SD)] errors between the contours produced by the proposed and conventional ASM models with respect to the manually delineated contours at the three different cross-sectional slices. The table shows the three error measures: MAD, Hausdorff distance, and Dice index. As can be seen in Table 2, the performance of the proposed ASM framework is better than that of the conventional ASM model. This is evident by the lower value of the MAD and Hausdorff measures and higher value of the Dice index.

The table also shows that the error of both methods is higher at the apical cross-sections compared with that at the basal and midcavity slices. This can be attributed to the increased blurring of the myocardium boundaries, due to the partial volume effects accentuated by the curvature of the RV FR wall near the apex. This results in a number of problems. First, it leads to increased operator uncertainty during the manual segmentation of the apical slices, which, in turn, leads to erroneous contours that do not properly capture the true shape of the RV. Another problem is that the appearance model is trained by vague intensity patterns that are not robust to be matched during the segmentation phase. It is worth noting that the performance of many other RV segmentation techniques was compromised at the apical level as reported by some researchers $[5,15]$.

Another observation that can be seen in Table 2, is that the errors at the basal slices are slightly higher than that at the midcavity slices. This is due to inaccurate delineation of some RV boundaries especially at end-systolic phases where the motion of the myocardial base causes parts of the RV boundaries to disappear (by moving towards the apex).

One advantage of the proposed ASM technique is its robustness especially in low quality images, where the RV boundaries are not sharp enough to attract the iterated ASM contour. This is because, at each iteration, the contour is implicitly constrained to pass through the two manually selected insertion points and thus maintains its correct position and pose. On the other side, the iterations of the conventional ASM, in absence of clear RV boundaries, could yield a contour of wrong pose and/or at wrong position.

The results of the pairwise t-test showed that the Hausdorff and the MAD errors of the proposed model are significantly lower than those of the conventional ASM. For basal, mid-cavity, and apical slices, a clear significant difference between the two methods was found $(p$-value $<0.005)$ for all comparing measures. Since the shape of the RV undergoes significant changes at the end-systole timeframe, all end-systole shapes in all cross-sectional slices have been separately tested. The results of these end-systole timeframes showed MAD, Hausdorf, and Dice indices of $4.1 \pm 2.4,8.0 \pm 4.5$, and $0.89 \pm 0.07$, respectively. Clearly, these values are within the average error range indicated in Table 2.

Fig. 5 shows the evolution of the ASM models from the initial contour to the contours at iterations number 5 and 20 for two patients. It can be seen in the figure that the initial contour of the proposed ASM framework is much better than that of the conventional ASM model. It is worth noticing that, as mentioned above, that both methods make use of the available manually selected RV insertion points. In fact, it was found that the initial and final errors of the conventional ASM model become worse if no rotation, translation, or scaling was applied. The figure also shows that the proposed ASM framework converges after almost 5 iterations whereas the conventional ASM model needs 15-20 iterations to correctly delineate the RV contour.

Case 1 in Fig. 5 shows the segmentation results for a patient with a dilated RV. As can be seen in the figure, both methods show approximately the same performance at the basal slice and almost converged at five iterations. For the mid-cavity and apical images, both methods resulted in accurate delineation of the FR part of the RV. Nevertheless, the proposed ASM method outperformed the conventional ASM at the SP part. This is mainly because of the dual nature of the proposed method where the FR contour segment can freely evolve to the correct RV boundaries independent of the SP segment. Fig. 5 shows the segmentation results of another case where the proposed ASM method shows results that are better than that of the conventional ASM especially at the apical slice.

Since the segmentation errors leads to estimation errors of the clinical parameters, we have calculated the ejection fraction of the RV and quantified the error, which was found to be 7.7 and $13.9 \%$ for the proposed ASM and the conventional ASM, respectively. The average computation times for segmenting one slice using a personal computer with dual-core $1.33 \mathrm{GHz}$ processor and $4 \mathrm{G}$ RAM were 0.09 and $0.17 \mathrm{~s}$ for the conventional and proposed ASM models, respectively. This increase in computation time in the proposed model is mainly due to the process of calculating the inverse Bookstein alignment equations. Nevertheless, the parallelised nature of the problem renders this difference insignificant. For example, several cardiac slices, as well as the two contour segments, can be simultaneously segmented using graphical processing units boards. Finally, it is worth noting that although the proposed framework extracts only the RV endocardium, the epicardium can be segmented through dilating the endocardium contour, similar to the work previously proposed in the literature [6].

\section{Conclusion}

In this work, a method for accurately segmenting the RV shape has been proposed. The method reduces the extraneous variations among the different RV shapes in the training dataset through two major steps: (i) splitting each RV contour into two simpler contour segments (SP and FR wall parts); and (ii) building a separate ASM model for each contour segment in the Bookstein coordinate space.

\begin{tabular}{|c|c|c|c|c|c|c|c|c|c|}
\hline & & MAD & & & Hausdorf & & & Dice index & \\
\hline & Basal & Mid & Apical & Basal & Mid & Apical & Basal & Mid & Apical \\
\hline & $4.0 \pm 3.5$ & $3.8 \pm 3.4$ & $4.0 \pm 4.3$ & $7.3 \pm 5.7$ & $6.8 \pm 5.2$ & $7.6 \pm 7.1$ & $0.91 \pm 0.07$ & $0.91 \pm 0.07$ & $0.86 \pm 0.11$ \\
\hline conventional ASM & $7.2 \pm 4.5$ & $6.0 \pm 3.3$ & $7.6 \pm 4.5$ & $11.2 \pm 6.9$ & $9.8 \pm 5.4$ & $12.8 \pm 7.0$ & $0.83 \pm 0.13$ & $0.84 \pm 0.14$ & $0.76 \pm 0.18$ \\
\hline
\end{tabular}

Table 2 Mean \pm SD of the MAD, Hausdorff, and Dice index measures of the segmented contours at basal, mid-cavity, and apical levels using the proposed and conventional ASM methods with respect to the ground truth 


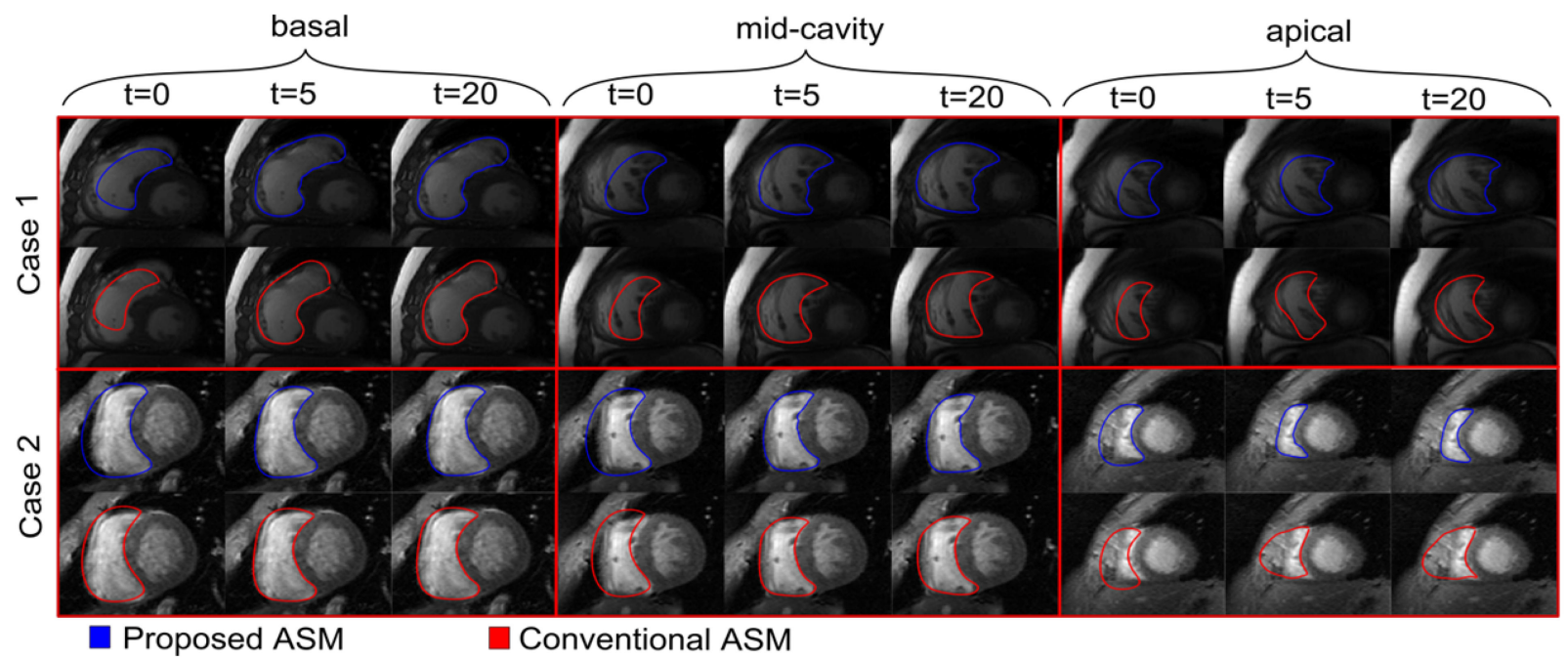

Fig. $5 R V$ segmentation results for two cases using the proposed and conventional ASM models at the initial, 5th, and 20th iterations. The figure shows three different cross-sections of the RV at the basal, mid-cavity, and apical levels

The results showed that the proposed method outperforms the conventional ASM method with more accurate results reached in less number of iterations. Future work includes investigating methods for automatic determination of the RV insertion points using intrinsic features, such as the contour curvature.

\section{Acknowledgment}

This work was fully funded by a research grant from the ITAC program, CFP \#59, ITIDA Agency, Ministry of Communication and Information Technology, Egypt.

\section{References}

[1] Voelkel, N.F., Quaife, R.A., Leinwand, L.A, et al.: 'Right ventricular function and failure: report of a national heart, lung, and blood institute working group on cellular and molecular mechanisms of right heart failure', Circulation, 2006, 114, (17), pp. 1883-1891

[2] Haddad, F., Hunt, S.A., Rosenthal, D.N., et al.: 'Right ventricular function in cardiovascular disease, part I: anatomy, physiology, aging, and functional assessment of the right ventricle', Circulation, 2008, 117, (11), pp. 1436-1448

[3] Haddad, F., Doyle, R., Murphy, D.J., et al.: 'Right ventricular function in cardiovascular disease, part II: pathophysiology, clinical importance, and management of right ventricular failure', Circulation, 2008, 117, (13), pp. $1717-1731$

[4] de Groote, P., Millaire, a., Foucher-Hossein, C., et al.: 'Right ventricular ejection fraction is an independent predictor of survival in patients with moderate heart failure', J. Am. Coll. Cardiol., 1998, 32, (4), pp. 948-954

[5] Grosgeorge, D., Petitjean, C., Caudron, J., et al.: 'Automatic cardiac ventricle segmentation in MR images: a validation study', Int. J. Comput. Assist. Radiol. Surg., 2011, 6, (5), pp. 573-581

[6] Wang, C., Peng, C., Chen, H.: 'A simple and fully automatic right ventricle segmentation method for 4-dimensional cardiac MR images'. RV Segmentation Challange in Cardiac MRI MICCAI, 2012

[7] Ou, Y., Doshi, J.: 'Multi-atlas segmentation of the cardiac MR right ventricle' Proc. 3D Cardiovascular Imaging: A MICCAI Segmentation Challenge, Nice, France, 2012

[8] Maier, O., Jiménez, D., Santos, A., et al.: 'Segmentation of RV in 4D Cardiac MR Volumes using Region-Merging Graph Cuts', in Computing in Cardiology (CinC), 2012, pp. 697-700

[9] Punithakumar, K., Noga, M., Ben Ayed, I., et al.: 'Right ventricular segmentation in cardiac MRI with moving mesh correspondences', Comput. Med. Imaging Graph., 2015, 43, pp. 15-25

[10] Mahapatra, D., Buhmann, J.M.: "Automatic cardiac RV segmentation using semantic information with graph cuts'. 2013 IEEE Tenth Int. Symp. on Biomedical Imaging, 2013, pp. 1106-1109

[11] Pluempitiwiriyawej, C.: 'Cardiac MR image segmentation: quality assessment of STACS'. IEEE Int. Symp. on Biomedical: Imaging Macro to Nano, 2004, (2), pp. 828-831

[12] Sacks, M.S., Chuong, C.J., Templeton, G.H., et al.: 'In vivo 3-D reconstruction and geometric characterization of the right ventricular free wall', Ann. Biomed. Eng., 1993, 21, (3), pp. 263-275

[13] Lorenzo-Valdés, M., Sanchez-Ortiz, G.I., Elkington, A.G., et al.: 'Segmentation of 4D cardiac MR images using a probabilistic atlas and the EM algorithm', Med. Image Anal., 2004, 8, (3), pp. 255-265

[14] Grosgeorge, D., Petitjean, C.: 'Graph cut segmentation with a statistical shape model in cardiac MRI', Comput. Vis. Image Underst., 2013, 117, (9), pp. $1027-1035$
[15] Petitjean, C., Zuluaga, M.A., Bai, W., et al.: 'Right ventricle segmentation from cardiac MRI: a collation study', Med. Image Anal., 2015, 19, (1), pp. 187-202

[16] Zhang, H., Wahle, A., Johnson, R.K., et al.: '4-D cardiac MR image analysis: left and right ventricular morphology and function', IEEE Trans. Med. Imaging, 2010, 29, (2), pp. 350-364

[17] ElBaz, M., Fahmy, A.: 'Active shape model with inter-profile modeling paradigm for cardiac right ventricle segmentation', Med. Image Comput Comput. Interv. - MICCAI, 2012, 7510, pp. 691-698

[18] van Ginneken, B., Frangi, A.F., Staal, J.J., et al.: 'Active shape model segmentation with optimal features', IEEE Trans. Med. Imaging, 2002, 21, (8), pp. 924-933

[19] Ordas, S., Boisrobert, L., Huguet, M., et al.: 'Active shape models with invariant optimal features (IOF-ASM) application to cardiac MRI segmentation'. Comput. Cardiol., 2003, pp. 633-636

[20] Lötjönen, J., Kivistö, S., Koikkalainen, J.: 'Statistical shape model of atria ventricles and epicardium from short-and long-axis MR images', Med. Image Comput. Comput. Interv. - MICCAI, 2004, 8, (3), pp. 371-386

[21] Frangi, A.F., Niessen, W.J., Viergever, M.A.: 'Three-dimensional modeling for functional analysis of cardiac images: a review', IEEE Trans. Med. Imaging, 2001, 20, (1), pp. 2-25

[22] Perperidis, D., Mohiaddin, R., Rueckert, D.: 'Construction of a 4D statistical atlas of the cardiac anatomy and its use in classification', Med. Image Comput. Comput. Interv. - MICCAI, 2005, 3750, pp. 402-410

[23] Huang, W., Goldgof, D.: 'Adaptive-size meshes for rigid and nonrigid shape analysis and synthesis', IEEE Trans. Pattern Anal. Mach. Intell., 1993, 15 (6), pp. 611-616

[24] Benayoun, S, Ayache, N.: 'Dense Non-Rigid Motion Estimation in Sequences of Medical Images Using Differential Constraints', Int. J. Comput. Vis., 1998, 26, (1), pp. 25-40

[25] Papademetris, X., Shi, P., Dione, D.: 'Recovery of soft tissue object deformation from 3D image sequences using biomechanical models', Inf. Process. Med. Imaging, 1999, 1613, pp. 352-357

[26] Shi, P., Sinusas, A.J., Constable, R.T., et al.: 'Point-tracked quantitative analysis of left ventricular surface motion from 3-D image sequences', IEEE Trans. Med. Imaging, 2000, 19, (1), pp. 36-50

[27] Friboulet, D., Magnin, I., Revel, D.: 'Assessment of a model for overall left ventricular three-dimensional motion from MRI data', Int. J. Card. Imaging, 1992, 8, (3), pp. 175-190

[28] Friboulet, D., Magnin, I., Mathieu, C.: 'Assessment and visualization of the curvature of the left ventricle from 3D medical images', Comput. Med. Imaging Graph., 1993, 17, (4-5), pp. 257-262

[29] Nastar, C., Ayache, N.: 'Frequency-based nonrigid motion analysis: Application to four dimensional medical images', IEEE Trans. Pattern Anal. Mach. Intell., 1996, 18, (11), pp. 1067-1079

[30] Clarysse, P., Friboulet, D., Magnin, I.E.: 'Tracking geometrical descriptors on 3-D deformable surfaces: application to the left-ventricular surface of the heart', IEEE Trans. Med. Imaging, 1997, 16, (4), pp. 392-404

[31] Matheny, A., Goldgof, D.: 'The use of three-and four-dimensional surface harmonics for rigid and nonrigid shape recovery and representation', IEEE Trans. Pattern Anal. Mach. Intell., 1995, 17, (10), pp. 967-981

[32] Staib, L.H., Duncan, J.S.: 'Model-based deformable surface finding for medical images', IEEE Trans. Med. Imaging, 1996, 15, (5), pp. 720-731

[33] Cootes, T., Taylor, C., Cooper, D., et al.: 'Active shape models-their training and application', Comput. Vis. Image Underst., 1995, 61, (1), pp. 38-59

[34] Cootes, T., Hill, A., Taylor, C. et al.: 'The use of active shape models for locating structures in medical images', Inf. Process. Med. Imaging, 2005, 687, pp. $33-47$

[35] El-Rewaidy, H., Fahmy, A.A.S.: 'Segmentation of the Right Ventricle in MR images using dual active shape model in the Bookstein coordinates'. 2015 IEEE 12th Int. Symp. on Biomedical Imaging (ISBI), 2015, pp. 1320-1323

[36] Hirose, K., Reed, J.E., Rumberger, J.A.: 'Serial changes in regional right ventricular free wall and left ventricular septal wall lengths during the first 4 
to 5 years after index anterior wall myocardial infarction', J. Am. Coll. Cardiol., 1995, 26, (2), pp. 394-400

[37] Leenders, G.E., Lumens, J., Cramer, M.J., et al.: 'Septal deformation patterns delineate mechanical dyssynchrony and regional differences in contractility: analysis of patient data using a computer model', Circ. Heart Fail., 2012, 5, (1), pp. 87-96

[38] Walmsley, J., Huntjens, P.R., Prinzen, F.W., et al.: 'Septal flash and septal rebound stretch have different underlying mechanisms'., Am. J. Physiol. Heart Circ. Physiol., 2015, 310, (3), pp. 394-403

[39] Brinkhoff, T., Kriegel, H., Schneider, R., et al.: 'Measuring the complexity of polygonal objects'. Proc. Third ACM SIGSPATIAL Int. Work. ACM Int. Work Advances in Geographic Information Systems, 1995, pp. 109-117

[40] Bookstein, F.: 'Size and shape spaces for landmark data in two dimensions', Stat. Sci., 1986, 1, (2), pp. 181-222
[41] Zhang, J.-Z., Xu, C.-X.: 'Trust region dogleg path algorithms for unconstrained minimization', Ann. Oper. Res., 1999, 87, (0), pp. 407-418

[42] Andreopoulos, A., Tsotsos, J.K.: 'Efficient and generalizable statistical models of shape and appearance for analysis of cardiac MRI', Med. Image Anal., 2008, 12, (3), pp. 335-357

[43] Babalola, K.O., Patenaude, B., Aljabar, P., et al.: 'An evaluation of four automatic methods of segmenting the subcortical structures in the brain', Neuroimage, 2009, 47, (4), pp. 1435-1447

[44] Huttenlocher, D.P., Klanderman, G.A., Rucklidge, W.J.: 'Comparing images using the Hausdorff distance', IEEE Trans. Pattern Anal. Mach. Intell., 1993, 15, (9), pp. 850-863

[45] Dice, L.R.: 'Measures of the amount of ecologic association between species', Ecology, 1945, 26, (3), pp. 297-302 